\title{
Contaminación en el Lago de Tota, Colombia: toxicidad aguda en Daphnia magna (Cladocera: Daphniidae) e Hydra attenuata (Hydroida: Hydridae)
}

July Andrea Barrera H. ${ }^{*}$, Adriana Janneth Espinosa R. ${ }^{2}$ \& Juan Pablo Álvarez S. ${ }^{3}$

1. Grupo de Investigación Unidad de Ecología en Sistemas Acuáticos. Universidad Pedagógica y Tecnológica de Colombia.Tunja, Colombia; andreba93@hotmail.com

2. Grupo de Investigación Unidad de Ecología en Sistemas Acuáticos. Universidad Pedagógica y Tecnológica de Colombia. Tunja, Colombia; adriana.espinosa@uptc.edu.co

3. Universidad Nacional de Colombia. Bogotá, Colombia; jpalvarezs@unal.edu.co

* Correspondencia

Recibido 07-VI-2017. Corregido 04-XI-2018. Aceptado 30-I-2019.

\begin{abstract}
Pollution in Lago de Tota, Colombia: acute toxicity on Daphnia magna (Cladocera: Daphniidae) and Hydra attenuata (Hydroida: Hydridae). Lago de Tota basin is currently being impacted by human activities like agriculture, deforestation, large-scale fish farming and monoculture of onion long (Allium fistulosum) with high use of agrochemicals and chicken manure that contaminate runoff to aquatic systems in the largest high mountain lake in Colombia. We implemented toxicity tests to evaluate possible deleterious effects of contaminants on H. attenuata and D. magna, model organisms commonly used in aquatic contamination assessment. Firstly, the organisms were acclimated and toxicity tests were validated in laboratory conditions. Later, water and sediments (elutriates) were evaluated from tributaries of Lago de Tota basin: Hato Laguna, El Tobal, Los Pozos, and Agua Blanca stream, during two contrasting hydroclimatic periods. In addition, pesticides in sediments were evaluated with chromatography, spectrometry and ultraviolet detection techniques. Acute toxicity tests showed that $H$. attenuata was more sensitive than D. magna, as it showed sublethal (Kruskal-Wallis; P $<0.05$ ) and lethal responses to exposure to waters and elutriates from Hato Laguna, El Tobal, and Los Pozos. At least five pesticides not quantified before in the basin were detected in chemical analyses (chlorothalonil, aldrin, dieldrin, DDT y congeners). These results indicate that anthropogenic activities have a potentially negative influence in aquatic communities composition, structure, and function, it's demand attention in future research.
\end{abstract}

Key words: Lago de Tota; Daphnia magna; Hydra attenuata; pesticides; sediments.

Barrera H., J. A., Espinosa R., A. J. \& Álvarez S., J. P. (2019). Contaminación en el Lago de Tota, Colombia: toxicidad aguda en Daphnia magna (Cladocera: Daphniidae) e Hydra attenuata (Hydroida: Hydridae). Revista de Biología Tropical, 67(1), 11-23.

Los ensayos de toxicidad acuática diagnostican el impacto de los contaminantes sobre la biota (Burton \& Ladrum, 2003; Blaise \& Gagné, 2009), al evaluar modificaciones en la fisiología normal de una población de organismos en un tiempo de exposición definido (Peluso, 2011). Evalúan los efectos agudos, subcrónicos o crónicos provocados por sustancias químicas presentes en las aguas crudas, residuales domésticas e industriales y agua de poro de sedimentos (Díaz, Bustos, \& Espinosa, 2004). Los ensayos agudos permiten calcular la concentración de una muestra que provoca la muerte al $50 \%$ de una población expuesta 
(concentración letal media o $\mathrm{CL}_{50}$ ) y/o la concentración que produce un determinado efecto en el $50 \%$ de la población (concentración efectiva media o $\mathrm{CE}_{50}$ ) en un tiempo conocido (Díaz et al., 2004; WHO \& IOMC, 2004).

El cladócero Daphnia magna Straus, 1820 y el pólipo de agua dulce Hydra attenuata Pallas, 1766 son aplicados en ecotoxicología gracias a la amplia distribución geográfica, pequeño tamaño, facilidad de cultivo, altas tasas de crecimiento poblacional (Sarma \& Nandini, 2006; Quinn, Gagné, \& Blaise, 2012) y sensibilidad a una gran variedad de sustancias tóxicas y mezclas, (Koivisto, 1995; Blaise \& Kusui, 1997). Los efectos tóxicos en D. magna se evidencian en alteraciones de tipo fisiológico y reproductivo, dificultades al nadar, alteración del ritmo cardíaco, inmovilidad y muerte. En $H$. attenuata los efectos son evidentes en los tentáculos, inician con aparición de bulbos en los extremos de estos, sigue en acortamiento, estado tulipán (fase avanzada de acortamiento de tentáculos) y finalmente desintegración (mortalidad) de los organismos (Blaise \& Kusui, 1997; Trottier, Blaise, Kusui, \& Johnson, 1997; Díaz et al., 2004; Quinn et al., 2012). A nivel latinoamericano se tienen avances en aplicación de pruebas de toxicidad en países como Brasil, México, Chile y Costa Rica. Para Colombia las pruebas de toxicidad son criterio de caracterización de residuos peligrosos y se cuenta con información en sustancias puras, mezclas de metales y vertimientos industriales, sin embargo, su aplicación en ambientes naturales es aún más escasa (Espinosa-Ramírez, 2018).

El lago de Tota es un sistema estratégico en los Andes Nororientales porque brinda agua para potabilizar, sostiene el desarrollo agropecuario e industrial de municipios como Sogamoso, Aquitania y otros (aproximadamente 250000 habitantes), recibe turismo por su belleza paisajística por ser el lago de alta montaña más grande del país. Ecológicamente es considerado centro de endemismos de vertebrados (Moncaleano \& Calvachi, 2009), importante para la conservación y migración de aves y la conservación de diversa biota acuática (CONPES 3801, 2014; Pedroza \& Rozo, 2017) que modula la estructura y función de este sistema.

El lago además soporta factores estresantes como la modificación del balance hídrico, la actividad piscícola industrial de trucha arcoíris (Oncorhynchus mykiss Walbaum, 1792), el vertimiento de aguas residuales domésticas generadas por tres municipios (Chaparro, 2013; CONPES 3801, 2014), junto con transformación paisajística por la duplicación del área de monocultivo de cebolla larga (Allium fistulosum), que cubre cerca de 2500 ha de su cuenca aferente y donde la aplicación de gallinaza cruda y agroquímicos es elevado (EspinosaRamírez, 2018). El lago presenta señales de un sistema ligeramente mesotrófico relacionado con descarga de nutrientes (por N), disminución de la transparencia en la columna de agua en los últimos quince años (Aranguren-Riaño et al., 2018) y aumento en la densidad de fitoplancton (Muñoz-López, Aranguren-Riaño, \& Duque, 2017) así como, por la expansión de litoral en sectores como Hato Laguna. Se reconoce que afluentes como El Tobal, Los Pozos y La Mugre, conducen productos de la escorrentía proveniente de las zonas agrícolas hacia la cubeta del lago (Cordero, Ruíz, \& Vargas, 2005; Noriega, Castillo, Vásquez, \& Monroy, 2010; Abella \& Martínez, 2012).

Respecto al ingreso de plaguicidas al lago, el conocimiento es más limitado a pesar de la larga tradición agrícola en la zona. Para el sector de Hato Laguna, se detectaron en 2009 en las aguas superficiales cuatro plaguicidas: malatión (insecticida), difenoconazol, tebuconazol y clorotalonilo (fungicidas) (Mojica \& Guerrero, 2013) en concentraciones bajas, pero con posibilidad de movilidad en los suelos de la región. En 2015 se recopiló información del uso de 183 agroquímicos para el cultivo de cebolla, con ingredientes activos como propineb, mancozeb y malatión entre los más usados (ver tablas I-III en Pérez, Grandas, Ramírez, \& Torres, 2016). Espinosa-Ramírez (2018), hace una revisión de la toxicología de algunos de los plaguicidas más utilizados y concluye la presencia de posibles agentes disruptores 
endocrinos, compuestos altamente móviles y persistentes, lo que indicaría una presión adicional por estas sustancias.

Debido a la importancia de este lago para la región y a la necesidad de seguir evaluando sus condiciones, el objetivo de esta investigación fue implementar ensayos biológicos con dos especies de amplio uso en pruebas estandarizadas de toxicidad (H. attenuata y $D$. magna) y aplicarlos para la detección de efectos tóxicos en muestras de agua y elutriados provenientes de la cuenca del lago de Tota ya que, hasta el momento, no se tiene información sobre posibles efectos deletéreos en el lago. Los resultados de esta investigación permitirán tener un primer acercamiento de la dinámica ecológica de los sistemas acuáticos frente a perturbaciones que afectan niveles de organización inferiores (individuos y poblaciones) y que repercutirían indirectamente sobre la comunidad plactónica, bentónica y nectónica y los diversos beneficios que brinda el lago a las comunidades humanas.

\section{MATERIALES Y MÉTODOS}

Aclimatación y mantenimiento organismos de prueba: El trabajo experimental se desarrolló en el laboratorio ECOTOX del grupo de investigación Unidad de Ecología en Sistemas Acuáticos (UDESA). Se propagaron los organismos (D. magna e H. attenuata) bajo condiciones controladas de variables ambientales, nutricionales y de crecimiento para obtener material biológico suficiente y con la calidad requerida para las pruebas de toxicidad con cloruro de sodio, muestras de agua y elutriados de sedimentos (Véase resultados de aclimatación en material suplementario).

Ensayos de toxicidad multi-concentración (sensibilidad): Los ensayos se realizaron con cloruro de sodio como tóxico de referencia (a partir de una disolución inicial de $10 \mathrm{~g} / 1 \mathrm{NaCl}$ ), debido a la intolerancia de los organismos de agua dulce a este compuesto y porque no genera residuos peligrosos para el ambiente. Con este procedimiento se identificó el intervalo de sensibilidad para los organismos de prueba y la concentración letal media $\left(\mathrm{CL}_{50}\right)$ que se usó como control positivo en ensayos con muestras del lago y elutriados (Véase sensibilidad en material suplementario).

Área de estudio: El lago de Tota es de origen tectónico-glacial, ubicado en el complejo de páramo Tota-Bijagual-Mamapacha (Morales et al., 2007) a 3015 m.s.n.m., con temperatura promedio de $11{ }^{\circ} \mathrm{C}$, profundidad máxima de $61 \mathrm{~m}$ y un volumen de aproximadamente 1920 millones de $\mathrm{m}^{3}$ de agua (Roldán \& Ramírez, 2008). El estudio se realizó en dos periodos climáticos contrastantes, para febrero 2016 (época de bajas precipitaciones) considerado por el Instituto de Hidrología, Meteorología y Estudios Ambientales (IDEAM, 2016) como el más extremo de la última década y abril (época de alta pluviosidad) con registros de $107 \mathrm{~mm}$ (Cañón-Barriga \& Valdés, 2011).

Se seleccionaron puntos en los tramos de la cuenca baja de cuatro afluentes, ya que en esas zonas la ronda del lago concentra las principales zonas de cultivo de cebolla larga (Allium fistulosum): Hato Laguna (3084 m.s.n.m., 05³5'08.7" N \& 72॰53'51.4" W), El Tobal (3036 m.s.n.m., 05³2'9.5' $\mathrm{N} \&$ 7253’21” W), Los Pozos (3048 m.s.n.m., 05'33'25.6” N \& 72 52'30.9' W) y Agua Blanca (3031 m.s.n.m., 05³0'58.9” N \& $\left.72^{\circ} 54^{\prime} 01.7^{\prime} \mathrm{W}\right)$. Con base en los resultados de toxicidad del primer muestreo se decidió tomar puntos adicionales en abril, con el propósito de obtener información de los denominados puntos de confluencia; sitio dentro del lago donde descarga el afluente, uno de ellos en el sector de La Custodia (3007 m.s.n.m., 05³0'54.5'" $\left.\mathrm{N} \& 72^{\circ} 54^{\prime} 23.3^{\prime \prime} \mathrm{W}\right)$ y los restantes en cercanía de las desembocaduras de los afluentes El Tobal, Los Pozos y La Mugre (Véase mapa área de estudio en material suplementario).

Muestreo de agua: Se recolectaron muestras puntuales en recipientes de vidrio de 11 para todos los sitios seleccionados $(n=12)$, se conservaron a una temperatura de $4{ }^{\circ} \mathrm{C}$ hasta su evaluación (ensayos de toxicidad aguda) dentro 
de las $36 \mathrm{~h}$ siguientes al muestreo (USEPA, 2001). Se tomaron además in situ variables física y químicas del agua como temperatura $\left({ }^{\circ} \mathbf{C}\right)$ y $\mathrm{pH}$ con $\mathrm{pH}$-metro Handylab pH11/SET, conductividad eléctrica $(\mu \mathrm{S} / \mathrm{cm})$ con conductímetro YSI 30, saturación de oxígeno (\%) con oxímetro YSI 55 y dureza $\left(\mathrm{mg} / 1 \mathrm{CaCO}_{3}\right.$, alcalinidad (mg/l $\left.\mathrm{HCO}_{3}\right)$, amonio $\left(\mathrm{mg} / 1 \mathrm{NH}_{4}\right)$, fosfatos $\left(\mathrm{mg} / \mathrm{PO}_{4-} \mathrm{P}\right)$ y nitratos $\left(\mathrm{mg} / \mathrm{NO}_{3}-\mathrm{N}\right)$ (Wetzel \& Likens, 2000) mediante Kits de Aqua-Merck.

Muestreo de sedimentos: Para establecer la acumulación de plaguicidas en sedimentos superficiales, se llevó a cabo muestreo por técnica de corazonador (Core PVC con $20 \mathrm{~cm}$ de longitud) en zonas de depositación, $\mathrm{y}$ en tres submuestras, que fueron mezcladas (Mejías \& Jerez, 2006; INECC-CCA, 2010; Pinedo, Marrugo, \& Díaz, 2015) y empacadas en material de vidrio protegido de la luz y refrigeradas en nevera para su transporte.

Para el análisis químico de plaguicidas se tomaron muestras en los cuatro afluentes elegidos en época de bajas precipitaciones y una muestra dentro del lago de Tota en el sector La Custodia, $(\mathrm{n}=5)$ se enviaron refrigeradas a laboratorio especializado para el análisis por el método multiresiduo para 359 componentes (plaguicidas organoclorados, organofosforados, carbamatos, piretroides y sus isómeros), con técnicas de cromatografía de gases y líquida seguida por detección mediante espectrometría de masas en tándem y detección ultravioleta para ditiocarbamatos con límites de cuantificación bajos.

Por otro lado, para las pruebas de toxicidad aguda con elutriados $(n=7)$ se tomaron muestras en Los Pozos y Agua Blanca para las dos épocas hidroclimáticas y en El Tobal, Hato Laguna y la confluencia Los Pozos en época de altas precipitaciones. Se utilizó la draga Eckman en los puntos de muestreo El Tobal y La Custodia dada su profundidad y sedimento fangoso.

Obtención de elutriados: Se obtuvieron los elutriados (extracto acuoso) a partir de los sedimentos recolectados, según protocolo de Pica y Trujillo (2008) donde se tomaron $100 \mathrm{~g}$ de sedimento con una paleta y se transfirieron a un recipiente de vidrio con una capacidad de $500 \mathrm{ml}$, éste se llenó hasta rebosar con agua potable embotellada y se sometió al baño ultrasónico (Branson 1516) durante 60 min evitando el sobrecalentamiento. Posteriormente se dejó decantar la muestra durante $12 \mathrm{~h}$ a temperatura de $4{ }^{\circ} \mathrm{C}$ y en completa oscuridad. Pasado el tiempo de reposo se procedió a extraer el sobrenadante (elutriado) en otro recipiente de vidrio, evitando resuspender el sedimento del fondo. El extracto obtenido se utilizó para la realización de los ensayos de toxicidad aguda, dentro de las $36 \mathrm{~h}$ siguientes al muestreo como lo sugiere la USEPA (2001). Se evaluaron parámetros fisicoquímicos al elutriado final (véase en material suplementario).

Ensayos de toxicidad en muestras del lago de Tota (uni-concentración, ensayos pasa-falla): Se realizaron tanto en muestras de agua $(n=12)$ como en elutriados $(n=7)$ con base en los protocolos descritos en Dutka (1989), Blaise y Kusui (1997) y Díaz et al. (2004). El diseño experimental consistió en un control positivo de $\mathrm{NaCl}$ (D. magna: $3.5 \mathrm{mg} / \mathrm{l}$ e H. attenuata: $1.0 \mathrm{mg} / \mathrm{l})$, un control negativo de agua embotellada y muestra de agua o elutriado en uni-concentración (100\% pasa o falla, muestra sin diluir, USEPA, 2001). Para $D$. magna el tiempo de exposición fue de $48 \mathrm{~h}$ y se utilizaron 21 neonatos (siete por replica) con menos de $24 \mathrm{~h}$ de nacidos, se contaron los individuos muertos a las 24 y $48 \mathrm{~h}$ para calcular la mortalidad (Dutka, 1989). Para H. attenuata el ensayo fue de $96 \mathrm{~h}$, se utilizaron nueve adultos sin yemas (tres por réplica), sin alimentación en $24 \pm 12 \mathrm{~h}$ previas al ensayo y se midieron efectos subletales (bulbo y cortos) y letales (tulipán y desintegrado) según protocolo de Blaise y Kusui (1997).

Tratamiento de datos: Los resultados de los ensayos de toxicidad con muestras de agua y elutriados se sometieron a la prueba ShapiroWilk, que permitió verificar el ajuste o no de los 
datos a una distribución normal. Se procedió a utilizar la prueba Kruskal-Wallis como alternativa no paramétrica al ANOVA de un factor. El propósito fue evaluar de manera independiente si factores como el punto de muestreo, el periodo hidroclimático (bajas precipitaciones y altas precipitaciones) y la matriz de los ensayos toxicológicos (agua y elutriados) afectaron significativamente las respuestas subletales y letales observadas. Los análisis se realizaron con el software Statgraphics Centurión XVII (López, 2013). Para las variables físicas y químicas se utilizó el análisis de componentes principales (ACP), para identificar patrones de variación temporal (dos periodos) y espacial (ocho puntos de muestreo); (McCune, Grace, \& Urban, 2002). El ACP se realizó con el software Canoco 4.5 (Lepš \& Šmilauer, 2003).

\section{RESULTADOS}

Aclimatación y mantenimiento organismos de prueba: Los organismos presentaron ciclo de vida acorde a lo determinado por literatura respecto a periodo de madurez sexual, en general, para D. magna transcurridos los primeros ocho días de vida las hembras alcanzaron su primer parto y se obtuvo una producción máxima de 145 a 180 neonatos, lo que permitió llevar a cabo las pruebas de toxicidad y la renovación de los cultivos sin dificultades. Igualmente, $H$. attenuata mantuvo condiciones fisiológicas normales y alta reproducción, el tiempo de duplicación a lo largo del periodo de estudio se mantuvo en dos días y se obtuvo una tasa de crecimiento $(\mathrm{K})$ de 0.35 , este valor refiere un crecimiento normal y condiciones fisiológicas óptimas para el desarrollo de pruebas de toxicidad según Trottier et al. (1997) y Díaz et al. (2004), quienes indican tasas para la especie entre 0.3 y 0.4 . Este seguimiento se dio durante los tres primeros meses para aprender a manejar y cultivar los organismos de prueba.

Variables físicas y químicas in situ (muestras de agua): Las diferencias en las variables medidas se presentaron entre épocas de muestreo más que a nivel espacial, como lo señala el análisis de componentes principales (ACP, Fig. 1). El componente 1 (50\%) y el 2 (23\%) explicaron el $73 \%$ de la varianza de los datos, la ordenación permitió distinguir

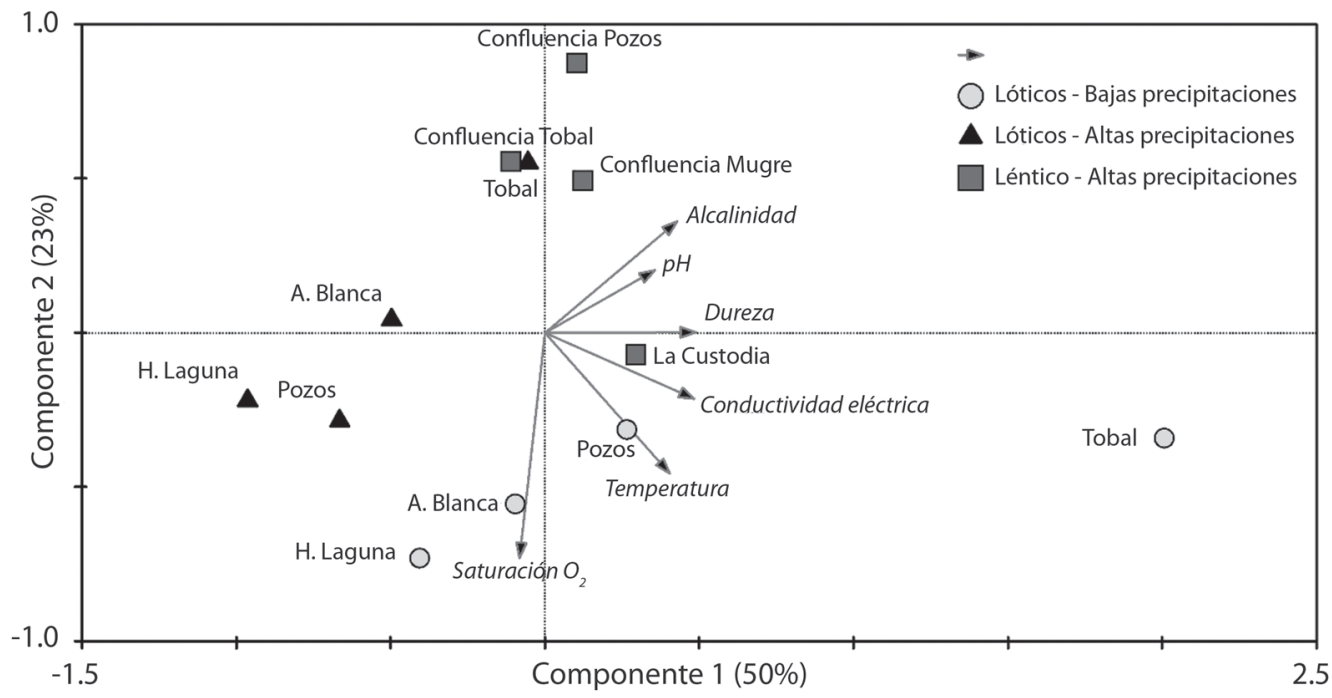

Fig. 1. Análisis de Componentes Principales (ACP), de las variables físicas y químicas teniendo en cuenta los sitios de muestreo dentro del lago de Tota (Boyacá, Colombia).

Fig. 1. Principal Component Analysis (PCA), physical and chemical variables and Lago de Tota (Boyacá, Colombia) sampling sites. 
que El Tobal se relacionó con altos niveles de conductividad y dureza, pero con bajos valores de saturación de oxígeno; Hato Laguna y Los Pozos se asociaron con mayores niveles de saturación de oxígeno, pero con bajos datos de conductividad y dureza; en época de altas precipitaciones todas las estaciones de los sistemas lóticos presentaron valores bajos para las variables medidas, al igual que la mayoría de los puntos de muestreo dentro del lago (lénticos). Espacialmente los afluentes El Tobal y Hato Laguna presentaron una mayor diferenciación en relación con los demás sitios.

\section{Ensayos de toxicidad aguda en muestras} del lago (uni-concentración, ensayos pasafalla): Los ensayos con D. magna para aguas, no evidenciaron respuestas positivas para ningún sitio. En $H$. attenuata, los ensayos agudos indicaron baja toxicidad, siendo Hato Laguna donde se obtuvo hasta un $66 \%$ de efectos subletales en bajas precipitaciones; respecto a letalidad, la máxima fue de $22 \%$ en Los Pozos, también en bajas precipitaciones. Las muestras de las confluencias indican baja toxicidad en la zona de mezcla (hasta $11 \%$ ) para ambos periodos evaluados (Cuadro 1). Estos resultados indican que los organismos fueron poco sensibles a los posibles contaminantes presentes en agua en los puntos evaluados, explicado quizá porque no están biodisponibles para los cladóceros e hidroideos, o se encuentran en concentraciones muy bajas para provocar un efecto letal.

Con respecto a los resultados de toxicidad con los elutriados $D$. magna tampoco presentó efectos de mortalidad a las $48 \mathrm{~h}$, mientras que $H$. attenuata, para época de bajas precipitaciones mostró un 17 y $61 \%$ de respuestas subletales para Agua Blanca y Los Pozos respectivamente. La época de altas precipitaciones mostró un $72 \%$ de respuestas subletales en El Tobal, en Los Pozos se presentó un $44 \%$, de igual manera la confluencia de Los Pozos mostró un $28 \%$, Hato Laguna tuvo un $17 \%$, finalmente Agua Blanca mostró un $11 \%$ de subletalidad (Cuadro 2), en todos los puntos la letalidad fue negativa o no fue mayor al $6 \%$.

Es apreciable que al evaluar con $H$. attenuata los elutriados, en todos los casos

\section{CUADRO 1}

Porcentaje de toxicidad en D. magna $(\mathrm{n}=12)$ e H. attenuata $(\mathrm{n}=12)$ para los dos periodos hidroclimáticos en muestras de agua

TABLE 1

Toxicity percentage D. magna $(\mathrm{n}=12)$ and $H$. attenuata $(\mathrm{n}=12)$ for both hydroclimatic periods in water samples

\begin{tabular}{|c|c|c|c|c|}
\hline \multirow{2}{*}{ Punto } & \multirow{2}{*}{ Época/Biomodelo } & \multirow{2}{*}{$\begin{array}{c}\text { D. magna } \\
\text { Mortalidad a } 48 \mathrm{~h}(\%)\end{array}$} & \multicolumn{2}{|c|}{ H. attenuata } \\
\hline & & & Subletalidad a 96 h (\%) & Letalidad a 96 h (\%) \\
\hline \multirow[t]{2}{*}{ Hato Laguna } & $\mathrm{BP}$ & $\mathrm{N}$ & 66 & 11 \\
\hline & AP & $\mathrm{N}$ & $\mathrm{N}$ & $\mathrm{N}$ \\
\hline \multirow[t]{2}{*}{ Los Pozos } & $\mathrm{BP}$ & $\mathrm{N}$ & $\mathrm{N}$ & 22 \\
\hline & AP & $\mathrm{N}$ & 6 & $\mathrm{~N}$ \\
\hline \multirow[t]{2}{*}{ El Tobal } & $\mathrm{BP}$ & $\mathrm{N}$ & 22 & $\mathrm{~N}$ \\
\hline & AP & $\mathrm{N}$ & $\mathrm{N}$ & $\mathrm{N}$ \\
\hline \multirow[t]{2}{*}{ Agua Blanca } & $\mathrm{BP}$ & $\mathrm{N}$ & 11 & $\mathrm{~N}$ \\
\hline & AP & $\mathrm{N}$ & $\mathrm{N}$ & $\mathrm{N}$ \\
\hline Confluencia Los Pozos & AP & $\mathrm{N}$ & $\mathrm{N}$ & $\mathrm{N}$ \\
\hline Confluencia El Tobal & AP & $\mathrm{N}$ & $\mathrm{N}$ & $\mathrm{N}$ \\
\hline Confluencia La Mugre & $\mathrm{AP}$ & $\mathrm{N}$ & 6 & $\mathrm{~N}$ \\
\hline La Custodia & AP & $\mathrm{N}$ & 11 & $\mathrm{~N}$ \\
\hline
\end{tabular}

*BP: Bajas precipitaciones; AP: Altas precipitaciones; N: negativo.

*BP: Low precipitations; AB: High precipitations; N: negative. 
CUADRO 2

Porcentaje de toxicidad en elutriados con D. magna $(\mathrm{n}=7)$ e H. attenuata $(\mathrm{n}=7)$ para los dos periodos hidroclimáticos

TABLE 2

Toxicity percentage in elutriates with D. magna $(\mathrm{n}=7)$ and H. attenuata $(\mathrm{n}=7)$ for both hydroclimatic periods

\begin{tabular}{lcccc}
\multicolumn{1}{c}{ Punto } & Época/Biomodelo & D. magna & \multicolumn{2}{c}{ H. attenuata } \\
\cline { 3 - 5 } Hato Laguna & Mortalidad $48 \mathrm{~h}(\%)$ & Subletalidad $96 \mathrm{~h}(\%)$ & Letalidad $96 \mathrm{~h}(\%)$ \\
Los Pozos & AP & $\mathrm{N}$ & 17 & 6 \\
& $\mathrm{BP}$ & $\mathrm{N}$ & 61 & $\mathrm{~N}$ \\
El Tobal & $\mathrm{AP}$ & $\mathrm{N}$ & 44 & 6 \\
Agua Blanca & $\mathrm{NP}$ & $\mathrm{N}$ & 72 & 22 \\
& $\mathrm{BP}$ & $\mathrm{N}$ & 17 & $\mathrm{~N}$ \\
Confluencia Los Pozos & $\mathrm{AP}$ & $\mathrm{N}$ & 11 & $\mathrm{~N}$ \\
\hline
\end{tabular}

*BP: Bajas precipitaciones; AP: Altas precipitaciones; N: negativo.

*BP: Low precipitations; AB: High precipitations; N: negative.

(Cuadro 2, $\mathrm{n}=7$ ) se cuantificaron principalmente efectos subletales, siendo El Tobal el que reporta adicionalmente efectos letales con hasta el $22 \%$ de los organismos expuestos. Se encontró mayor toxicidad en ambos periodos hidroclimáticos en Los Pozos, explicado probablemente porque tienen bajo caudal $\left(0.25 \mathrm{~m}^{3} / \mathrm{s}\right.$; CONPES $3801,2014)$ y amplia afectación por los cultivos de cebolla en ambas laderas. Los análisis estadísticos señalan que el factor matriz (aguaelutriados) influye significativamente sobre la subletalidad de $H$. attenuata, especialmente los elutriados (Kruskal-Wallis, $\mathrm{P}=0.006 ; \mathrm{P}<0.05$ ), con un nivel de confianza del $95 \%$.

Identificación de plaguicidas en sedimentos: El Cuadro 3 reporta la presencia de plaguicidas en tres de los sitios de muestreo evaluados. El sitio con mayor número de plaguicidas fue Los Pozos donde se encontraron DDT y congéneres, aldrín, dieldrin, ditiocarbamatos (maneb, mancozeb, metiram, propineb, thiram y ziram) y clorotalonilo. En segundo lugar, estuvo Hato Laguna con presencia de los mismos ditiocarbamatos correspondientes a los plaguicidas de mayor uso actualmente según lo reportado por Mojica y Guerrero (2013) y Pérez et al. (2016). Los sedimentos para El Tobal y La Custodia (alta precipitación) fueron negativos a la presencia de plaguicidas.

\section{DISCUSIÓN}

Para el desarrollo de este trabajo se cultivaron los organismos de prueba en agua embotellada, que presentó una dureza baja (45.6 mg/l CaCO3, $\mathrm{n}=3$ ) comparada con lo sugerido para el cultivo de D. magna (160-180 $\mathrm{mg} / \mathrm{l} \mathrm{CaCO} 3)$, se modificó esta variable frente a lo recomendado por otros autores debido a que el agua del lago de Tota presenta características de aguas blandas (Gil, Pedroza, \& Aranguren, 2016). Aunque Cowgill y Milazzo (1991) afirman que el éxito reproductivo de esta especie puede verse afectada por dureza menor a 72 $\mathrm{mg} / \mathrm{CaCO}_{3}$, Terra y Feiden (2003) encontraron que $D$. magna no modifica el comportamiento o la reproducción a diferentes niveles de dureza $\left(10-250 \mathrm{mg} / 1 \mathrm{CaCO}_{3}\right)$ fenómeno similar al encontrado en este trabajo y que permitió el correcto desempeño de los ensayos de sensibilidad al cloruro de sodio, con muestras de agua y elutriados provenientes del lago.

Las pruebas con $H$. attenuata se han considerado adecuadas para la evaluación de toxicidad (Blaise \& Kusui, 1997) y fueron más sensibles para las aguas y elutriados en afluentes del lago de Tota comparado con D. magna, el organismo de prueba estándar en ensayos de toxicidad. Los resultados indicarían que en el agua evaluada hay bajas concentraciones de contaminantes que no afectan la fisiología de 
CUADRO 3

Plaguicidas detectados en sedimentos en microcuencas del Lago de Tota

TABLE 3

Pesticides in sediments in microbasins of Lago de Tota

\begin{tabular}{|c|c|c|c|c|}
\hline Sistema Lótico & Resultado & $\begin{array}{l}\text { Límite de reporte / } \\
\text { método }(\mathrm{mg} / \mathrm{kg})\end{array}$ & $\begin{array}{l}\text { Concentración } \\
(\mathrm{mg} / \mathrm{kg})\end{array}$ & Interpretación \\
\hline Hato Laguna & $\begin{array}{l}\text { Ditiocarbamatos, Maneb, mancozeb, } \\
\text { metiram, propineb, thiram y ziram. }\end{array}$ & $0.05 / \mathrm{UV}$ & 0.12 & $\begin{array}{l}\text { Uso reciente encuesta } \\
2009-2013\end{array}$ \\
\hline \multirow[t]{4}{*}{ Los Pozos } & $\begin{array}{l}\text { Ditiocarbamatos, Maneb, mancozeb, } \\
\text { metiram, propineb, thiram y ziram. }\end{array}$ & $0.05 / \mathrm{UV}$ & 0.17 & $\begin{array}{l}\text { Uso reciente encuesta } \\
2009-2013\end{array}$ \\
\hline & Aldrin y Dieldrin & $\begin{array}{l}0.02 \text { y } 0.01 / \\
\text { GC-MSMS }\end{array}$ & 0.014 & Uso Prohibido \\
\hline & Clorotalonilo & 0.01 / GC-MSMS & 0.017 & $\begin{array}{l}\text { Uso reciente encuesta } \\
2009-2013\end{array}$ \\
\hline & DDT y congéneres & 0.01 / GC-MSMS & 0.082 & Uso Prohibido \\
\hline El Tobal & & & $\leq \mathrm{LC}$ & \\
\hline Agua Blanca & DDT y congéneres & 0.01 / GC-MSMS & 0.012 & Uso Prohibido \\
\hline La Custodia & & & $\leq \mathrm{LC}$ & \\
\hline
\end{tabular}

* CG-MS MS: cromatografía de gases acoplada a masas-masas; UV: detección ultravioleta para ditiocarbamatos en bajos niveles; LC: Límite de cuantificación. DDT y congéneres: (suma de p,p'-DDT, o,p'-DDT, p-p'-DDE y p,p'-TDE (DDD) expresado como DDT).

* GC-MS MS: gas chromatography coupled to masses-masses; UV: ultraviolet detection for dithiocarbamates at low levels; LC: Limit of quantification. DDT and congeners: (sum of p,p'-DDT, o,p'-DDT, p-p '-DDE and p,p' -TDE (DDD) expressed as DDT).

D. magna e H. attenuata a nivel agudo. Los litorales del lago cercanos a la desembocadura de los afluentes presentan macrófitas que desempeñarían un papel biodepurador ya que absorben parte de los nutrientes contaminantes que drenan al cuerpo de agua (Corpoboyacá, 2005; Secretaría de Salud Boyacá, 2012) y que aplicaría también para otros contaminantes, lo que podría relacionarse con el bajo porcentaje de respuestas letales y subletales que se obtuvieron en los ensayos de toxicidad en aguas. Brogan y Relyea (2014), revelan que el aumento del $\mathrm{pH}$ por el proceso de fotosíntesis de las macrófitas promueve la hidrólisis del malatión lo cual evita la absorción por parte de D. magna de este contaminante, y aunque estos mecanismos no se pueden generalizar para todos los plaguicidas aplicados en la zona, si podrían explicar parcialmente la baja sensibilidad de esta especie a los plaguicidas detectados en la cuenca. La movilidad y persistencia de los plaguicidas dependen de su estabilidad química y de su afinidad con partículas de suelo o agua.
Investigaciones con $H$. attenuata confirman que la mezcla de contaminantes en agua puede actuar de forma aditiva y provocar efectos subletales como los cambios morfológicos reportados, y sugieren que el aumento en las concentraciones ambientales de estos contaminantes puede resultar posiblemente en efectos negativos para otros taxones acuáticos (Quinn et al., 2007, 2008), lo que se podría reflejar en El Tobal y Los Pozos donde se presentaron las mayores respuestas tóxicas para la especie, igualmente podría explicarse por la presencia de los plaguicidas en este último sitio (Cuadro 3). Demetrio, Rossini, Bonetto, y Ronco (2012), al evaluar plaguicidas provenientes de cultivos de soya en Argentina con Hydra, determinaron que es muy sensible a herbicidas, mientras en esta investigación se encontró que fungicidas e insecticidas son los más utilizados en la cuenca del lago de Tota y podrían estar implicados en los efectos observados.

La presencia de aldrin, dieldrin, DDT y congéneres en sedimentos de los afluentes 
(Cuadro 3) representan una preocupación por ser considerados compuestos orgánicos persistentes, caracterizados por potencialidad de bioacumulación y toxicidad, además, son frecuentes en sedimentos de sistemas acuáticos en Brasil y en algunas regiones de Norteamérica (Venkatesan et al., 2010; Hunt et al., 2016). Estos compuestos son insecticidas organoclorados con potencial disrupción endocrina en humanos y altamente lipofílicos, lo que los hace bioacumulables y mediante procesos de biomagnificación son movilizados por las redes tróficas (Garrison et al., 2014). Sus residuos pueden persistir en el ambiente por periodos prolongados (7-30 años) (Yu, Tsunoda, \& Tsunoda, 2011). Esta persistencia, podría explicar la presencia en la cuenca del lago ya que desde 1986 está prohibido su uso en Colombia. Al encontrar estos residuos se constituye una alerta porque podrían afectarse significativamente la fauna acuática del lago, fenómeno que debe evidenciarse en futuros trabajos de investigación.

El área de estudio tiene una amplia tradición agrícola, antes de la expansión del monocultivo de cebolla larga, puesto que se producía también papa (Solanum tuberosum; 600 ha en áreas de ladera y quebradas), arveja (Pisum sativum; 90 ha en Tota y Cuítiva), trigo (Triticum spp; 75 ha), cebada (Hordeum vulgare; 38 ha), zanahoria (Daucus carota; 10 ha) y haba (Vicia faba; 22 ha en Tota) (CONPES 3801, 2014). La agricultura tradicional supone presión por el uso intensivo de plaguicidas en la cuenca, lo cual explicaría los hallazgos en sedimentos y/o implicaría que han sido depositados desde otras regiones, ya que actualmente se acepta que las altas montañas son zonas de deposición de compuestos orgánicos que se movilizan por corrientes de aire (Catalan, Ninot, \& Aniz, 2017) fenómeno poco explorado en los sistemas acuáticos colombianos.

La extracción de elutriados y los análisis de toxicidad permitieron desprender los contaminantes asociados al sedimento y brindar información sobre la biodisponibilidad de los mismos (Domene, Alcaniz, \& Andrés, 2008), los resultados señalan respuestas subletales en los dos períodos muestreados y que aunque la letalidad estuvo por debajo del $20 \%$ (baja) se observó en época de altas precipitaciones, lo que indicaría la posible movilidad de fracciones contaminantes del suelo que se depositan y resolubilizan en sedimentos. Aunque los extractos acuosos no representan con exactitud la exposición observada en sedimentos enteros (Harkey, Landrum, \& Klaine, 1994), son un valioso complemento a las pruebas de fase sólida (Nebeker et al., 1984).

Las sustancias tóxicas retenidas por el sedimento pueden ser resuspendidas por actividades humanas como el dragado (Sprovieri et al., 2007), práctica evidente en la cuenca del lago de Tota ya que se realiza almacenamiento y depósito en canales para riego. Es posible que la condición química de los elutriados pueda explicar las respuestas biológicas obtenidas, fenómeno que debe profundizarse en investigaciones futuras también con la medición de plaguicidas en esta solución. Aunque las concentraciones de plaguicidas detectadas en sedimentos fueron bajas, no es posible descartar que los efectos letales y subletales obtenidos en $H$. attenuata en El Tobal, Los Pozos y Hato Laguna se deban a su presencia. La interacción de los plaguicidas con otras sustancias junto con factores ambientales como la temperatura, tipo de suelo y actividad bacteriana, debe considerarse cuando se llevan a cabo pruebas para la evaluación de efectos deletéreos, ya que contribuyen en la variación de la toxicidad (Gardner, 1979; Nowell, Norman, Ingersoll, \& Moran, 2016).

No se encontraron plaguicidas en El Tobal, probablemente porque en este punto se dificultó la extracción de sedimento por la profundidad en el sector escogido para el muestreo, se reconoce que esta microcuenca es la más extensa de la cuenca del lago de Tota, con un área de 3048 ha y 2990 predios dedicados al cultivo de cebolla, además, confluyen aproximadamente doce quebradas que abarcan una extensa zona de la cuenca (CONPES 3801, 2014). Se aclara que la recolección de una sola muestra no es representativa para este sector, por lo tanto, un resultado negativo debe interpretarse 
con precaución. Con base en los resultados de plaguicidas se recomienda ampliar el muestreo de los mismos en agua, suelos y sedimentos, dado que cinco muestras no son representativas para las dinámicas de uso agrícola en la cuenca, aunque indican que tres de cada cinco muestras dieron positivo para uno o más plaguicidas, lo cual debe motivar un mayor seguimiento a estos agentes.

Se reconoce que la probabilidad de detectar plaguicidas y su variabilidad espacial y temporal en cuerpos de agua superficiales depende de las condiciones ambientales locales, a la frecuencia de aplicación en la zona (Mojica \& Guerrero, 2013) así como a las características químicas propias del plaguicida. Para la cuenca del lago se considera una frecuencia de uso alta, ya que se aplican cada ocho días en época de altas precipitaciones y cada quince días en época seca (Mojica \& Guerrero, 2013). Las concentraciones de plaguicidas en las corrientes de agua se consideran efímeras y se sugiere monitorear la presencia y permanencia en el agua, los sedimentos y principalmente en fauna, de aquellos organismos con hábitos bentónicos como los macroinvertebrados que se consideran sensibles a estas modificaciones ambientales (Schäfer et al., 2011).

Otro aspecto importante a tener en cuenta en la zona estudiada es la proximidad residencial con las actividades agrícolas, en otras regiones del mundo por esta exposición se ha demostrado anomalías en humanos, como bajo peso al nacer (Xiang, Nuckols, \& Stallones, 2000), muerte fetal (Bell et al., 2001) y cánceres infantiles (Reynolds et al., 2002). Se sugiere fortalecer la vigilancia epidemiológica a los residentes en la cuenca pues el uso intensivo de plaguicidas puede afectar la salud humana incluso a las futuras generaciones por presencia de disruptores endocrinos como los plaguicidas clorotalonilo, aldrin, dieldrin y DDT. Estos compuestos son sustancias exógenas capaces de alterar el equilibrio hormonal, actúan a dosis muy bajas, se producen trastornos de tipo reproductivo, en el desarrollo de órganos y tejidos, así como alteraciones en los procesos metabólicos (Bergman et al., 2013).

Dada la baja respuesta con las pruebas de toxicidad aguda (D. magna e H. attenuata), es necesario explorar afectaciones a largo plazo en comunidades hidrobiológicas nativas e iniciar ensayos de toxicidad crónica con cladóceros, monitoreo in situ con microalgas, pruebas de bioacumulación y/o biomarcadores con $O$. mykiss (trucha arcoíris) y en los tributarios con macroinvertebrados y perifiton, dados los hallazgos en los ensayos con elutriados y con la presencia confirmada de diversos plaguicidas en la microcuenca de los Pozos y Hato Laguna. La problemática ambiental percibida en las microcuencas hace necesario anotar que pueden estar ingresando microcontaminantes no monitoreados ampliamente a esta fuente de agua estratégica para la región. Este trabajo es uno de los primeros en centrar la atención sobre efectos de los plaguicidas en esta cuenca $\mathrm{y}$ se espera en investigaciones futuras ampliar el entendimiento sobre este factor estresor no contemplado previamente.

Declaración de ética: los autores declaran que todos están de acuerdo con esta publicación y que han hecho aportes que justifican su autoría; que no hay conflicto de interés de cualquier tipo; y que han cumplido con todos los requisitos y procedimientos éticos y legales pertinentes. El documento firmado se encuentra en los archivos de la revista.

\section{AGRADECIMIENTOS}

A la Dirección de Investigaciones (DIN), por el apoyo financiero en la convocatoria Capital Semilla 2015 de la Universidad Pedagógica y Tecnológica de Colombia. A la Universidad Nacional de Colombia, laboratorio de Ingeniería Ambiental por ceder el material biológico al grupo de investigación Unidad de Ecología en Sistemas Acuáticos (UDESA) y a las diversas personas que contribuyeron en este trabajo. 


\section{RESUMEN}

La cuenca del Lago de Tota ha sido modificada por diversas actividades humanas, como deforestación, piscicultura y el monocultivo de cebolla larga (Allium fistulosum) con un elevado uso de agroquímicos y gallinaza cruda, causando deterioro de las características ecológicas del sistema léntico de alta montaña más grande de Colombia. En este trabajo se implementaron pruebas de toxicidad para determinar posibles efectos deletéreos sobre H. attenuata y D. magna. Se aclimataron los organismos y validaron los ensayos de toxicidad en condiciones de laboratorio, posteriormente se realizó la evaluación de agua y sedimentos (elutriados) provenientes de afluentes de la cuenca del lago: Hato Laguna, El Tobal, Los Pozos y Agua Blanca, durante dos periodos hidroclimáticos contrastantes. Se evidenció una mayor sensibilidad con los ensayos en $H$. attenuata, ya que señalan que aguas y elutriados de Hato Laguna, El Tobal y Los Pozos generaron respuestas de subletalidad (Kruskal-Wallis; P $<0.05$ ) y letalidad. Se registra presencia positiva de al menos cinco plaguicidas no cuantificados antes en la cuenca (clorotalonilo, aldrin, dieldrin, DDT y congéneres). Estos resultados indicarían una potencial influencia negativa de las actividades antropogénicas sobre organismos acuáticos nativos, con posibles consecuencias sobre la composición, estructura y función de las comunidades hidrobiológicas.

Palabras clave: Lago de Tota; Daphnia magna; Hydra attenuata; plaguicidas; elutriados.

\section{REFERENCIAS}

Abella, J. P., \& Martínez, M. J. (2012). Contribución de un afluente tributario a la eutrofización del lago de Tota (Boyacá, Colombia). Revista Colombiana de Quimica, 41(2), 243-261.

Aranguren-Riaño, N. J., Shurin, J. B., Pedroza-Ramos, A., Muñoz-López, C. L., López, R., \& Cely, O. (2018). Sources of nutrients behind recent eutrophication of Lago de Tota, a high mountain Andean lake. Aquatic Sciences, 80(4), 39.

Bell, E. M., Hertz-Picciotto, I., \& Beaumont, J. J. (2001). A case-control study of pesticides and fetal death due to congenital anomalies. Epidemiology, 12(2), 148-156.

Bergman, Å., Heindel, J. J., Jobling, S., Kidd, K., Zoeller, T. R., \& World Health Organization. (2013). State of the science of endocrine disrupting chemicals 2012. World Health Organization.

Blaise, C., \& Gagné, F. (2009). Bioassays and biomarkers, two pillars of ecotoxicology: past, present and prospective uses. Fresenius Environmental Bulletin, 18(2), 135-139.
Blaise, C., \& Kusui, T. (1997). Acute toxicity assessment of industrial effluents with a microplate-based Hydra attenuata assay. Environmental Toxicology, 12, 53-60.

Brogan III, W. R., \& Relyea, R. A. (2014). A new mechanism of macrophyte mitigation: How submerged plants reduce Malathion's acute toxicity to aquatic animals. Chemosphere, 108, 405-410.

Burton, A., \& Landrum, F. (2003). Toxicity of sediments. In G. V. Middleston, M. J. Church, M. Corigilo, L. A. Hardie, \& F. J. Longstaffe (Eds.), Encyclopedia of Sediments and Sedimentary Rocks (pp. 748-751). Dordrecht: Kluwer Academic Publishers.

Catalan, J., Ninot, J. M., \& Aniz, M. M. (2017). The high mountain conservation in a changing world. In High Mountain Conservation in a Changing World (pp. 3-36). Springer, Cham.

Cañón-Barriga, J. E., \& Valdés, J. (2011). Assessing the influence of global climate and anthropogenic activities on the water balance of an Andean Lake. Journal of Water Resource and Protection, 3, 883-891.

Chaparro, J. (2013). Cambios institucionales para preservar la cantidad y calidad del agua en la cuenca de Lago de Tota, Colombia (Tesis de Maestría). Pontificia Universidad Javeriana, Bogotá.

CONPES 3801-Consejo Nacional de Política Económica y Social. (2014). Manejo ambiental integral de la cuenca hidrográfica del Lago de Tota. República de Colombia. Recuperado de: http://veetota.weebly. com/conpes-3801-lago-de-tota.html

Cordero, R. D., Ruiz, J. E., \& Vargas, E. F. (2005). Determinación espacio-temporal de la concentración de fósforo en el lago de Tota. Revista Colombiana de Química, 34(2), 211-218.

Corpoboyacá - Corporación Autónoma Regional de Boyacá. (2005). Plan de ordenación y manejo de la cuenca del lago de Tota, Caracterización Fisicoquímica del Recurso Hídrico, Capítulo IV-B. Recuperado de: http://www.corpoboyaca.gov.co/cms/wp-content/ uploads/2015/11/diagnostivo-caracterizacion-fisicoquimica-lago-tota.pdf

Cowgill, U. M., \& Milazzo, D. P. (1991). Demographic effects of salinity, water hardness and carbonate alkalinity on Daphnia magna and Ceriodaphnia dubia. Archiv für Hydrobiologie, 122(1), 35-56.

Demetrio, P. M., Rossini, G. D. B., Bonetto, C. A., \& Ronco, A. E. (2012). Effects of pesticide formulations and active ingredients on the coelenterate Hydra attenuata (Pallas, 1766). Bulletin of Environmental Contamination and Toxicology, 88(1), 15-19.

Díaz-Báez, M. C., Bustos-López, M. C., \& EspinosaRamírez, A. J. (2004). Pruebas de Toxicidad acuática: Fundamentos y métodos. Bogotá, D.C: Editorial UNIBIBLOS, Universidad Nacional de Colombia. 
Domene, X., Alcaniz, J. M., \& Andrés, P. (2008). Comparison of solid-phase and eluate assays to gauge the ecotoxicological risk of organic wastes on soil organisms. Environmental Pollution, 151(3), 549-558.

Dutka, B. J. (1989). Methods for microbiological and toxicological analysis of waters, wastewaters and sediments. Ontario, Canada: National Water Research Institute (NWRI), Canada Centre of Inland Water.

Espinosa-Ramírez, A. J. (2018). El agua, un reto para la salud pública. La calidad del agua y las oportunidades para la vigilancia en Salud Ambiental (Tesis Doctoral). Universidad Nacional de Colombia, Bogotá.

Gardner, D. E. (1979). Introductory remarks: Session on genetic factors affecting pollutant toxicity. Environmental Health Perspectives, 29, 45-48.

Garrison, W., Cyterski, M., Roberts, K. D., Burdette, D., Williamson, J., \& Avants, J. K. (2014). Occurrences and fate of DDT principal isomers/metabolites, DDA, and $\mathrm{o}, \mathrm{p}$ '-DDD enantiomers in fish, sediment and water at a DDT-impacted superfund site. Environmental Pollution, 194, 224-234.

Gil, L. N., Pedroza, A. X., \& Aranguren, N. J. (2016). Valoración ambiental del litoral del lago de Tota basado en estructura y función de macroinvertebrados. Cultura Cientifica, 14, 16-25.

Harkey, G. A., Landrum, P. F., \& Klaine, S. J. (1994). Comparison of whole-sediment, elutriate and pore-water exposures for use in assessing sediment-associated organic contaminants in bioassays. Environmental Toxicology and Chemistry, 13(8), 1315-1329.

Hunt, L., Bonetto, C., Resh, V. H., Buss, D. F., Fanelli, S., Marrochi, N., \& Lydy, M. J. (2016). Insecticide concentrations in stream sediments of soy production regions of South America. Science of the Total Environment, 547, 114-124.

INECC-CCA - Instituto Nacional de Ecología y Cambio Climático-Centro de Ciencias de la Atmósfera. (2010). Manual de métodos de muestreo y preservación de muestras de las sustancias prioritarias para las matrices prioritarias del Proname. México.

IDEAM - Instituto de Hidrología, Meteorología y Estudios Ambientales. (13 de enero de 2016). El Niño continúa en su fase de mayor intensidad. Grupo de comunicaciones IDEAM. Bogotá, D.C. Recuperado de: http:// www.ideam.gov.co/web/sala-de-prensa/noticias.

Koivisto, S. (1995). Is Daphnia magna an ecologically representative zooplankton species in toxicity tests? Environmental Pollution, 90(2), 263-267.

Lepš, J., \& Šmilauer, P. (2003). Multivariate analysis of ecological data using CANOCO. Cambridge University Press.
López, C. P. (2013). Análisis Multivariate de Datos: Aplicaciones con IBM SPSS, SAS y STATGRAPHICS. Garceta.

McCune, B., Grace, J. B., \& Urban, D. L. (2002). Analysis of Ecological Communities (Vol. 28). Gleneden Beach, OR: MjM software design.

Mejías, J., \& Jerez, J. (2006). Guía para la toma de muestras de residuos de plaguicidas: agua, sedimento y suelo. Santiago de Chile: Instituto de Investigaciones Agropecuarias. Centro Regional de Investigación Carillanca.

Mojica, A., \& Guerrero, J. A. (2013). Evaluación del movimiento de plaguicidas hacia la cuenca del lago de Tota. Colombia. Revista Colombiana de Química, 42(2), 29-38.

Moncaleano, Á. M., \& Calvachi, B. A. (2009). Uso de la fauna silvestre del Lago de Tota. Peces, herpetos, aves y mamíferos. Ambiente y Desarrollo, 13(25), 81-99.

Morales, M., Otero, J., Van der Hammen, T., Torres, A., Cadena, C., Pedraza, C., ... Cárdenas, L. (2007). Atlas de páramos de Colombia. Bogotá, D. C.: Instituto de Investigación de Recursos Biológicos Alexander von Humboldt.

Muñoz-López, C. L., Aranguren-Riaño, N. J., \& Duque, S. R. (2017). Functional morphology of phytoplankton in a tropical high mountain lake: Tota Lake (BoyacaColombia). Revista de Biología Tropical, 65(2), 669-683.

Nebeker, A. V., Cairns, M. A., Gakstatter, J. H., Malueg, K. W., Schuytema, G. S., \& Krawczyk, D. F. (1984). Biological methods for determining toxicity of contaminated freshwater sediments to invertebrates. Environmental Toxicology and Chemistry, 3(4), 617-630.

Noriega, J., Castillo, D., Vásquez, A., \& Monroy, J. (2010). Estudio preliminar del estado de la microcuenca Los Pozos y su posible efecto en un sector de la laguna de Tota, Boyacá-Colombia. Revista de Tecnología, 9(2), 145-164.

Nowell, L. H., Norman, J. E., Ingersoll, C. G., \& Moran, P. W. (2016). Development and application of freshwater sediment-toxicity benchmarks for currently used pesticides. Science of the Total Environment, $550,835-850$.

Pedroza, R. A., \& Rozo, S. S. (2017). Catálogo de la biota acuática del lago de Tota (Boyacá-Colombia): Fitoplancton, Zooplancton y Zoobentos. Tunja: Editorial UPTC.

Peluso, M. (2011). Evaluación de los efectos biológicos y biodisponibilidad de contaminantes en sedimento del Río de La Plata y afluentes (Tesis Doctoral). Universidad Nacional de la Plata, Argentina. 
Pérez, W. J., Grandas, I. A., Ramírez, L. N., \& Torres, N. H. (2016). Identification of the main active ingredients of agrochemicals used around the Lake of Tota, Colombia. Revista Ciencia y Agricultura, 13(1), 91-106.

Pica, G., \& Trujillo, G. (2008). Procedimiento para la generación de extractos orgánicos y elutriados de suelos y sedimentos para su análisis en ensayos de toxicidad. En P. Ramírez \& A. Mendoza (Eds.), Ensayos Toxicológicos para la Evaluación de Sustancias Químicas en Agua y Suelo (pp. 371-382). México: Instituto Nacional de Ecología.

Pinedo, J., Marrugo, J., \& Díaz, S. (2015). Speciation and bioavailability of mercury in sediments impacted by gold mining in Colombia. Chemosphere, 119, 1289-1295.

Quinn, B., Gagné, F., \& Blaise, C. (2007). Validation of a multi-well plate toxicity test to assess feeding behaviour of the cnidarian, Hydra attenuata. Fresenius Environmental Bulletin, 16(9), 1100-1107.

Quinn, B., Gagné, F., \& Blaise, C. (2008). An investigation into the acute and chronic toxicity of eleven pharmaceuticals (and their solvents) found in wastewater effluent on the cnidarian, Hydra attenuata. Science of the Total Environment, 389(2), 306-314.

Quinn, B., Gagné, F., \& Blaise, C. (2012). Hydra, a model system for environmental studies. The International Journal of Development Biology, 56, 613-625.

Reynolds, P., Von Behren, J., Gunier, R. B., Goldberg, D. E., Hertz, A., \& Harnly, M. E. (2002). Childhood cancer and agricultural pesticide use, an ecologic study in California. Environmental Health Perspectives, 110(3), 319-324.

Roldán, G., \& Ramírez, J. J. (2008). Fundamentos de Limnología neotropical ( $2^{\text {nd }}$ ed.). Medellín: Editorial Universidad de Antioquia.

Sarma, S. S. S., \& Nandini, S. (2006). Review of Recent Ecotoxicological Studies on Cladocerans. Journal of Environmental Science and Health, Part B, 41(8), 1417-1430.

Schäfer, R. B., Pettigrove, V., Rose, G., Allinson, G., Wightwick, A., Von der Ohe, P. C., ... Kefford, B. J. (2011). Effects of pesticides monitored with three sampling methods in 24 sites on macroinvertebrates and microorganisms. Environmental Science Technology, 45(4), 1665-1672.

Secretaría de Salud Boyacá. (2012). Mapa de riesgo de la calidad del agua para consumo humano de la laguna de Tota, fuente abastecedora del casco urbano de los municipios de Firavitova y Cuitiva. Recuperado de: http://www.boyaca.gov.co/SecSalud/direcciones/ dirección-de-salud-pública/calidad-agua-consumohumano/414-mapas-de-riesgo-de-salud-ambiental

Sprovieri, M., Feo, M. L., Prevedello, L., Manta, D. S., Sammartino, S., Tamburrino, S., \& Marsella, E. (2007). Heavy metals, polycyclic aromatic hydrocarbons and polychlorinated biphenyls in surface sediments of the Naples harbour (Southern Italy). Chemosphere, 67(5), 998-1009.

Terra, N. R., \& Feiden, I. R. (2003). Reproduction and survival of Daphnia magna Straus, 1820 (Crustacea: Cladocera) under different hardness conditions. Acta Limnologica Brasiliensia, 15(2), 51-55.

Trottier, S., Blaise, C., Kusui, T., \& Johnson, E. M. (1997). Acute toxicity Assessment of Aqueous Samples Using a Microplate-based Hydra attenuata Assay. Environmental Toxicology, 12, 265-271.

USEPA - United States Environmental Protection Agency. (2001). Methods for collection, storage and manipulation of sediments for chemical and toxicological analyses: Technical Manual EPA 823-B-01-002. Washington, D. C.

Venkatesan, M. I., Merino, O., Baek, J., Northrup, T., Sheng, Y., \& Shisko, J. (2010). Trace organic contaminants and their sources in surface sediments of Santa Monica Bay, California, USA. Marine Environmental Research, 69(5), 350-362.

Wetzel, R., \& Likens, G. (2000). Limnological Analyses $\left(2^{\text {nd }}\right.$ ed.). New York: Springer Science + Business Media.

WHO \& IOMC - World Health Organization and InterOrganization Programme for the Sound Management of Chemicals. (2004). IPCS risk assessment termino$\log y$ (Vol. 1). World Health Organization.

Xiang, H., Nuckols, J. R., \& Stallones, L. A. (2000). Geographic information assessment of birth weight and crop production patterns around mother's residence. Environmental Research, 82(2), 160-167.

Yu, M. H., Tsunoda, H., \& Tsunoda, M. (2011). Environmental toxicology: biological and health effects of pollutants ( $3^{\text {rd }}$ ed.). Boca Ratón, London, New York: CRC Press, Taylor \& Francis Group.

See Digital Appendix at: / Ver Apéndice digital en:

revistas.ucr.ac.cr 